\title{
Comparative study of the physical and tensile properties of Jicama (Pachyrhizus erosus) starch film prepared using three different methods
}

\begin{abstract}
The objective of this work is to study the physical and tensile properties of jicama (Pachyrhizus erosus) starch film prepared using three different methods. First, a film is prepared from starch granules after sifting using a sieve shaker. A second film is prepared from starch granules after ultra-sonication. Another film is made by sonicating the starch gel. Ultrasonication is performed using an ultrasonic probe. These three different methods have a significant effect on the properties of the film ( $\mathrm{p} \cdot 0.05)$. The film from the starch granules after sifting using $63 \mu \mathrm{m}$ mesh size and ultrasonication (labeled as S-63U film) shows the optimum properties. Opacity for S-63U film is almost half $(48.6 \%)$ that of the equivalent non-sonicated film. S-63U film has the highest tensile strength $(3.1 \mathrm{MPa})$, the lowest moisture absorption (18\% after $8 \mathrm{~h}$ in a humid chamber) and water vapor permeability. FESEM morphology of the fracture surface of the sonicated film display a more homogeneous structure compared to films without ultrasonication.
\end{abstract}

Keyword: ANOVA; Bioplastics; Starch; Ultrasonication 